\title{
Novel Framework for Breast Cancer Classification for Retaining Computational Efficiency and Precise Diagnosis
}

\author{
Vidya K. \\ Research scholar, Siddartha Academy of Higher \\ Education, Tumkur, India
}

\author{
Kurian M. Z., PhD \\ Registrar, Siddartha Academy of Higher Education, \\ Tumkur, India
}

\begin{abstract}
Classification of breast cancer is still an open-end challenge in medical image processing. The existing literatures were reviewed to found that existing solution are more pivotal towards accuracy in classification and less towards achieving computational effectiveness in classification process. Therefore, this paper presents a novel classification approach that bridges the trade-off between computational performances of classifier with its final response towards disease criticality. An analytical framework is built that takes the input of Magnetic Resonance Imaging (MRI) of breast cancer which is subjected to non-linear map-based filter for enhancing pre-processing operation. The algorithm also offers a novel integral transformation scheme that lets the filtered image to get itself transformed followed by precise extraction of foreground and background for assisting in reliable classification. A statistical-based approach is used for extracting feature followed by classifying using unsupervised learning algorithm. The study outcome shows superior performance compared to existing schemes of classification.
\end{abstract}

\section{Keywords}

Breast Cancer, Classification, MRI Image, Malignant/ Benign, Classifier

\section{INTRODUCTION}

With the advance of medical image processing in terms of soft-computing and hardware-based techniques, there has been a significant progress in the area of non-invasive disease diagnosis in case of critical condition of carcinoma [1]. According to highly reliable sources, it was found that approximately 252, 710 members have been reported for invasive breast cancer and 63, 410 members are found to suffer from in-situ breast cancer in the present year of 2017 [2]. This is a very simple current statistics to show that breast cancer is one alarming disease condition where still medical science is lagged behind for performing an efficient detection of it in early stage [3]. Although, there are some good number of work carried out towards cancer detection [4][5], but still there is no reported cases to claim $100 \%$ successful identification owing to various causes associated with capturing of radiological images. Therefore, this problem offers a significant impediment towards exploring better possibility of performing classification of breast cancer. Basically, such forms of classification is carried out on the basis of multiple schemes, where each schemes uses discrete criteria and are used for different problems. However, the significant factors in classification are prominent grade of tumor, form of histopathological, current stage of tumor, etc, which is more or less clinical in nature [6]. The target of performing an effective and precise classification is to assists in precise determination of treatment as minor mistake in cancer classification may lead to wrong treatment at the cost of life of patient. Hence accuracy is highly demanded. At present, there are various forms of classification algorithms already used in medical image processing [7][8][9][10]; however, there are significant level of problems in all these classification types and approaches. The first problems is associated with pre-processing, where majority of the existing studies have less focused on. Usually, Magnetic Resonance Imaging (MRI) images have higher possibilities of having multiplicative noises, which are required to be controlled. However, there are also possibilities of different other forms of noises, Hence, it is really an expensive affair to develop a universal technique to address any form of noise problems in medical image processing. The second problem is related to mechanism of processing the image in order to retain maximum information. The existing mechanism actually doesn't process the input image but it subjects it to some algorithm that directly assists in classification. Because of this phenomenon, the image looses much significant information that affects in detection as well as classification steps. This problems leads to another problem that is associated with computational effectiveness for which reason the system can only perform linear classification and very less non-linear classification. The presence of such problems is not novel and existed from long time back. Therefore, the proposed study presents an implementation plan of a novel classification technique which essentially focuses on computational effectiveness and then on accuracy level. The system uses true positive and false positive as the prime parameters to measure accuracy from multiple observations. Sub-Section 1.1 discusses about the existing literatures where different techniques of classification are discussed for breast cancer followed by discussion of research problem in Sub-Section 1.2 and proposed solutionin Sub-Section 1.3. Algorithm implementation is discussed in Section 2 followed by result discussion in Section 3. Section 4 summarizes the paper contribution.

\subsection{Background}

This section discusses about the techniques adopted for performing classification of disease condition pertaining to medical image dataset. Ashraf et al. [11] have used markov random modeling for performing classification of MRI image of breast cancer. Most recently, the work done by Beevi et al. [12] have used multi-classifier using deep neural network for same purpose. Beura et al. [13] have used transform based on discrete orthonormal for assisting in disease detection. Usage of multiscale morphology was seen in the work done by Chen et al. [14] for solving classification problems. Adoption of trace transform-based methodology was seen in the work 
carried out by Ganesan et al. [15] for classification of mammograms. Odajima and Pawlovsky [16] have used KNN algorithm for diagnosis of breast cancer. Pawlovsky and Nagahashi [17] have enhanced the KNN performance further to increase the mean accuracy of breast cancer diagnosis. Uniyal et al. [18] have used time-series based analysis for predicting the likelihood of malignancy. Literatures have also witnessed a major adoption of Artificial Neural Network towards solving the similar purpose of breast cancer classification. Ahmad et al. [19] have combinely used genetic algorithm and neural network with better accuracy performance. Usage of only neural network towards solving similar problem was witnessed in the work done by Jouni et al. [20]. Kanojia and Abraham [21] have used radial-basis function for performing detection of histopathological images of breast cancer. Usage of feed-forward as well as back propagation was investigated by Saini and Vijay [22] toward analyzing mammograms. Similar trend of solution was also seen in the work carried out by Solanki et al. [23] with an uniqueness of adding synthetic noise for exploring more about falsification in alarms. Spanhol et al. [24] have used convolution neural network for where the performance of feed-forward has further been enhanced to solve the classification problems. The work carried out by Utomo et al. [25] have used gradient-based back propagation algorithm using extreme learning technique for assisting in solving diagnosis problems. Neural network was also used for localizing the critical position of tumour in breast as seen in the study of Wahab et al. [26]. Apart from neural network, KNN algorithm was also found to be frequently used in literatures for similar causes. Alpaslan et al. [27] have used $\mathrm{KNN}$ algorithm for classifying masses of breast cancer. Hybridization of KNN algorithm with support vector machine was seen in the work of Bouazza et al. [28] towards solving classification problems in gene expression. The potential of KNN algorithm was also found to be used for identifying abnormalities on brain MRI images as seen in the work of Hachemi et al. [29]. Machhale et al. [30] have also integrated support vector machine with $\mathrm{KNN}$ algorithm for mitigating the classification problems in brain MRI images. Therefore, it can be seen that there are various level of work being carried out towards addressing classification problems in radiological images. However, the summary of findings are i) less work towards classification is carried out on breast MRI image, ii) neural network and $\mathrm{KNN}$ is frequently practiced methods for solving classification problems, iii) less focus on computational efficiency and more focus on accuracy was seen. Hence, such problems do exists in existing literature inspire of its advantageous points towards classification. The next section discusses about the identification of research problem followed by brief highlights of the adoption research methodology as a solution to solve the identified research problem.

\subsection{Problem Identification}

The problems to be addressed in presented scheme are as follows:

- Although MRI images generates superior radiological image, but its interpretation is highly subjective that gives rise to variability in clinical conclusion about the disease among different observers.

- No automated technique to perform identification and classification of stages of carcinoma for a given MRI data.
- Even with high resolute MRI, existing research works are still witnessing false positives even in identification stages.

- Existing techniques are found to have more focus on accomplishing accuracy and less towards computational effectiveness of the classification process.

Therefore the problem statement of the proposed study can be defined as "To develop a single cost effective modeling that can address both classification problems in breast cancer and simulatenously retain computational efficiency for breast MRI image."

\subsection{Proposed Solution}

The prime purpose of the proposed study is to present a novel classification approach in order to distinguish malignant and benign condition using integral transformation scheme. The main purpose of this scheme is to apply integral transformation scheme to design and develop a novel set of classifier in screening breast cancer. The significant contribution of this scheme is to develop a simple analytical model using integral transformation scheme in order to perform reliable extraction of feature from the MRI data followed by classification. Usage of integral transformation scheme is rare to find in existing research implementation of screening / classifying breast cancer criticality. This part of the study will consider adopting a simple analytical-based approach in methodology formulations. The study will consider investigating from the The Cancer Imaging Archive (TCIA) [31] etc. The initial part of the study will check for the pre-processing operation to be carried out, which is expected to make the image suitable for classification.

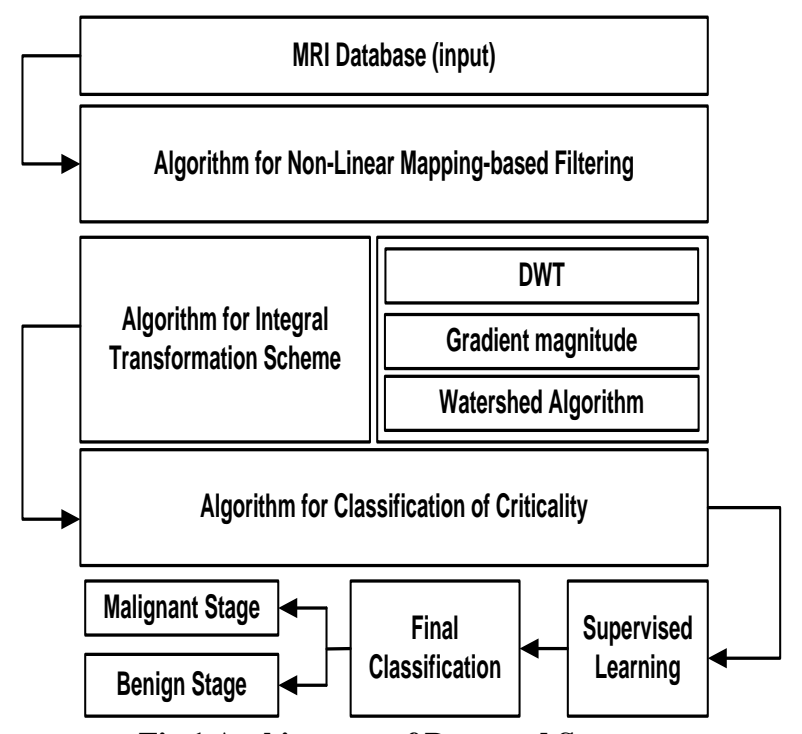

Fig 1 Architecture of Proposed System

The significant contribution to investigate for possible computational complexity associated with implementing integral transformation scheme. It is because the integral is basically an operation to hold uniform absolute value in the traversing direction of tracing vector with a constraint of considering the vector only once. But in order to solve the computational problem, this part of the work will need to have the vector to go around two different directions in order to ensure zero impact of variability. A simple analytical formulation will be carried out for these purposes that can significant assists in extracting potential statistical features. 
The next part of the study will be to develop a novel classifier that can perform better distinction. The approach will also use supervised learning technique for better compatibility with both linear and non-linear classification feasibility. A probabilistic distribution model will be used for enabling computational tractability. The final outcome of the model will lead to generation of classification of breast MRI image to be malignant or benign. The next section discusses algorithm implementation.

\section{ALGORITHM IMPLEMENTATION}

This section discusses about the algorithm implementation and design for the purpose of classifying the malignant or benign stage of the tumor from the MRI image of the breast cancer. During the implementation of the algorithm, an emphasis was laid over the algorithm's computational effectiveness apart from the classification accuracy. It is because the primary motive of the algorithm is to incorporate higher degree of robustness which will mean that the algorithm will offer similar performance of detection / classification irrespective of the quantity of the MRI image of breast cancer. It is because incorporation of such functionalities will render higher scale of applicability in real-time scenario. The discussion of the core algorithms are as follows:

\subsection{Algorithm for Non-Linear Mapping- based Filtering}

This algorithm is mainly responsible to increase the eligibility criteria of MRI image towards better classification process owing to the complexities related to the form of the medical image. The algorithm takes the input of an MRI image I in DICOM format (Line-1) in order to ensure that it adheres to real-time nature of MRI image exercised in the area of diagnostics. From the input image, the respective information about number of rows $r$ and columns $c$ is estimated (Line-1) and stored in one master matrix A. The next step of the algorithm is to perform preliminary enhancement of the input image by managing both contrast as well as brightness of an input image in order to address the problem of multiplicative noise that occurs frequently in MRI image. A nested loop is created to read all the rows as well as columns to finally formulate the master matrix $A$ which essentially forms a nonlinear mapping operation as shown in Line-4. The non-linear mapping attribute $\mathrm{H}$ is finally extracted as shown in Line-5. The attribute $\mathrm{H}$ is further upgraded for normalizing the different forms of frequency components for better detectability (Line-8). The algorithm performs approximate localization of the frequency domain followed by considering Logarithm of the intensity image (Line-9) as the reflectance as well as illumination integrates together. Therefore, it is feasible for segregate multiplicative components linearly over the frequency domain. Fast Fourier Transform operation is carried out on the top of it (Line-9). It has to be understood that the algorithm applies logarithm function in order to ensure that Fast Fourier Transform could be applied It is because of the fact that it is very much challenging task to implement Fast Fourier Transform as the elementary form of the master matrix doesn't represent any form of product equation. This problem was addressed by applying logarithm on it that is further followed by Fast Fourier Transform (Line-9). For better effectiveness, the technique maximizes the high frequency components $\alpha \mathrm{H}$ and minimizes the low frequency components $\alpha \mathrm{L}$. It is because generally the reflectance is represented with an aid of high frequency $\alpha \mathrm{H}$ and components of lower frequency are represented by $\alpha \mathrm{L}$. Therefore, the system applies high pass filtering for minimizing the minimal frequency components and enhances the maximized frequency components.

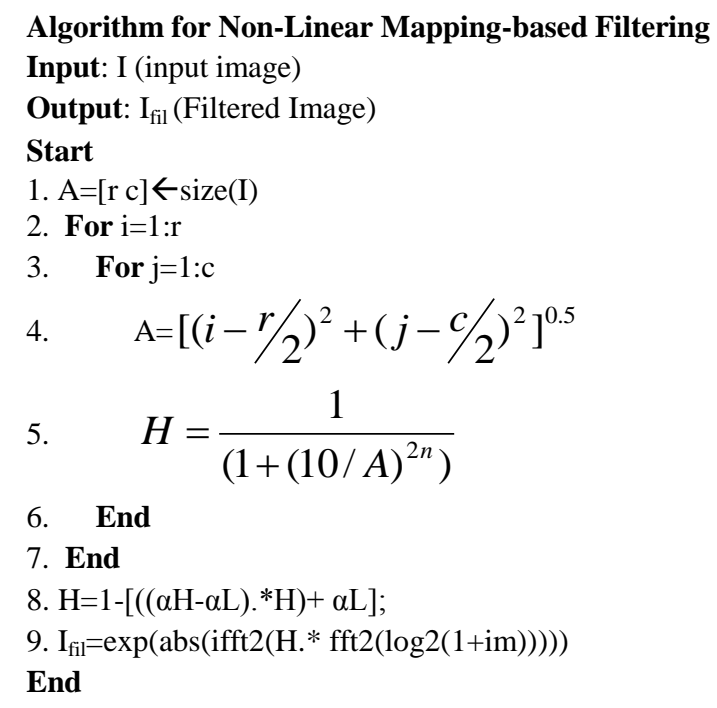

Therefore, the above mentioned algorithm enhances the illumination factor of the MRI image using its filtering operation with an aid of non-linear maps that can control both brightness and contrast at same time. The outcome of the algorithm is a filtered image $I_{\text {fil }}$ obtained from inverse Fast Fourier Transform (Line-9), which is further subjected to next algorithm of integral transformation scheme.

\subsection{Algorithm for Integral Transformation Scheme}

This algorithm is mainly responsible for applying a novel logic of integral transformation scheme which will mean that the algorithm should make the input image further more compatible to be performing the classification. Hence, this algorithm can be also said to be direct contribution towards enhancing the classification and detection scheme of proposed system. Basically, the algorithm is meant for integration a transformation as well as segmentation scheme together for obtained better compatible image. The steps of the algorithm are as shown below:

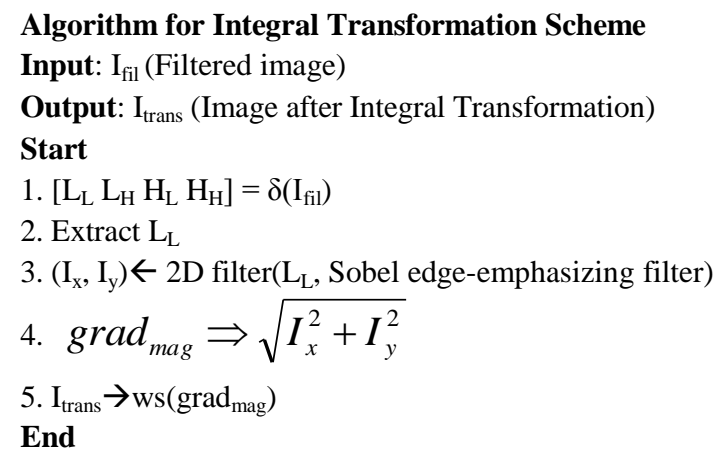

The output of the first algorithm i.e. filtered image $\mathrm{I}_{\text {fil }}$ acts as input for this algorithm, which is further subjected to two dimensional discrete wavelet transformation using Haar wavelets $\delta$ (Line-1). The implication of this preliminary transformation leads to generation of 4 different coefficients e.g. $\mathrm{L}_{\mathrm{L}}$ (low-Low), $\mathrm{L}_{\mathrm{H}}$ (Low-High), $\mathrm{H}_{\mathrm{L}}$ (High-Low), and $\mathrm{H}_{\mathrm{H}}$ (High-High). This step is followed by extraction of only $\mathrm{L}_{\mathrm{L}}$ coefficient for further implementation in algorithm steps (Line2 ). The next step of the algorithm is to apply a two dimensional filter of pre-defined form where Sobel operator is used in order to emphases the edge during the filtering process. Therefore, the proposed algorithm offers increasing emphasis on the edge- 
based information that will significant assist in determining the stage of classification in the next phase of algorithm implementation. Therefore, the algorithm applies two dimensional filters and stores it in a temporary matrix say hy and also extracts the transform of hy to store it in $h x$ i.e. $h x=h y^{\mathrm{T}}$. Further two dimensional filters are applied on both hy and $\mathrm{hx}$ in order to obtain $I x$ and $I y$ (Line-3). Finally, the system computes the gradient magnitude rad $_{\text {mag }}$ as shown in Line-4. The next stage of the algorithm is to apply watershed algorithm to the gradient magnitude obtained in the previous step (Line5). This step is also followed by further smaller operations e.g. i) indexing the foreground objects, removal of dark spots (to address false positives), applying maximum image region to extract better foreground information, cleaning of the edges and elimination of blobs with less pixel information. Similarly, the backgrounds are also indexed followed by applying watershed algorithm in order to obtain better version of segmented image. Therefore, this algorithm results in a finally transformed image $I_{\text {trans }}$ which is not only transformed by discrete wavelet transformation but also segmented using watershed algorithm on its gradient magnitude obtained.

\subsection{Algorithm for Classification of Criticality}

This is the last stage of the algorithm design and implementation where the prime motive of this algorithm is to perform classification of the criticality of the stages of the tumor of the breast cancer from the MRI image. It is essential that this algorithm should maintain increased amount of accuracy in the detection process. As imprecise diagnosis of disease will also lead to selection of imprecise treatment by the physician therefore, it is necessary to maintain higher degree of accuracy and precision. Therefore, the proposed system doesn't directly design or apply classification algorithm on the input image but does some further steps to ensure that more information be extracted from the input image. The input image of this algorithm is basically the output obtained from prior algorithmic step i.e. $I_{\text {trans. For extracting more }}$ information, the proposed study obtains multiple statistical information. Various descriptive statistical parameters e.g. S(standard deviation), K (Kurtosis), M (Mean), Sk (skewness), $\mathrm{V}$ (variance), M2/M3/M4 (Moments) are extracted and stored in a matrix $\mathrm{F}$ which cumulatively records all the features (Line1). The next part of the study is to apply Support Vector Machine on the extracted feature F (Line-2). An operator $o p$ was used for storing all the iterations of the classification process using unsupervised learning approach (Line-2-3). As the proposed system uses unsupervised learning approach to perform classification which has supportability for both linear as well as non-linear classification approach therefore, the anticipated value of op is always 1 for benign case of tumor. However, if op is found equivalent to 2 than the algorithm infers it to be a presence of a malignant tumor (Line-4-9). The steps involved in the proposed algorithm are as follows:

\footnotetext{
Algorithm for Classification of Criticality deviation), K (Kurtosis), M (Mean), Sk (skewness), V (variance), M2/M3/M4 (Moments)

Output: Classify_outcome

Start

1. $\mathrm{F}=\left[\mathrm{S}, \mathrm{K}, \mathrm{M}, \mathrm{Sk}, \mathrm{V}, \mathrm{M} 2, \mathrm{M} 3, \mathrm{M} 4,{ }^{\prime} \mathrm{I}_{\text {trans }}{ }^{\prime}\right]$

2. op $\rightarrow \operatorname{svm}(\mathrm{F})$

3. op $=\operatorname{round}\left(\mathrm{op}^{\prime}\right)$

4. If $\mathrm{op}==2$

5. $\quad$ tumorflag $=1$;

6. End

7. If tumorflag $==1$
}

Input: $\mathrm{I}_{\text {trans }}$ (Image after Integral Transformation), S(standard
8. Classify_outcome $\rightarrow$ Detected Tumor 9. End

End

The primary contribution of the proposed classification technique is that it is nearly similar to existing optimization based algorithms that uses evolutionary-based approaches. Interestingly, the design of the proposed classification algorithm is also free from any predefined dataset as well as it is highly scalable irrespective of the sizes of the datasets. The next section discusses about the result accomplished from the proposed study.

\section{RESULT ANALYSIS}

The evaluation of the proposed system is carried out considering the DICOM format of the images taken from Cancer Imaging Archive [31]. All the images are normally grayscale in the dataset, which are initially subjected to be converted in the form of matrix using Matlab environment. Fig. 2 shows some of the visual outcome where input image after being subjected to the algorithmic steps results in the generation of the finally transformed image. The cases of benign identification can be seen in Fig.2(a) as input image, Fig.2(b) as transformed image with emphasis on edges, and Fig.2(c) on final identification of zero tumor. Similarly, identification of malignancy can be seen in Fig.2(d) as input image, Fig.2(e) as transformed image, while Fig.2(f) as finally identification of tumour in malignancy stage.

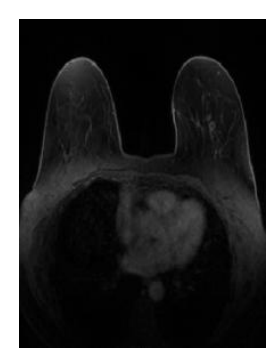

(a)

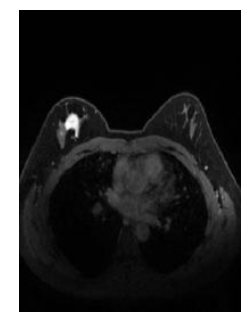

(d)

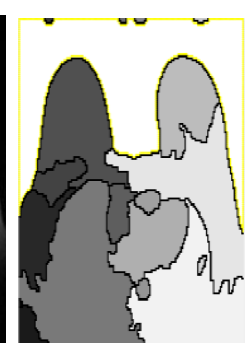

(b)

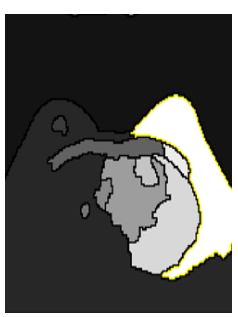

(e)

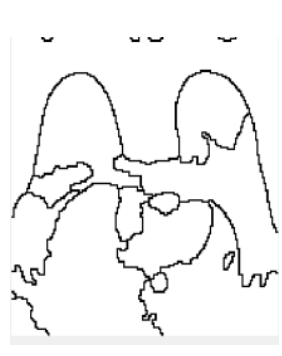

(c)

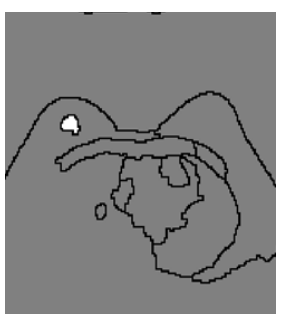

(f)
Fig 2 Visual Outcomes of Proposed System

The outcome of the proposed study was also compared with the more frequently used neural network and KNN algorithm. For this purpose, the framework continues the similar steps till integral transformation scheme for existing system with the last step of classification being performed separately by feedforward network (in neural network) and KNN algorithm in order to maintain similar test-bed environment for comparative performance analysis. The outcomes are assessed with respect to true positive and false positive and is shown below 


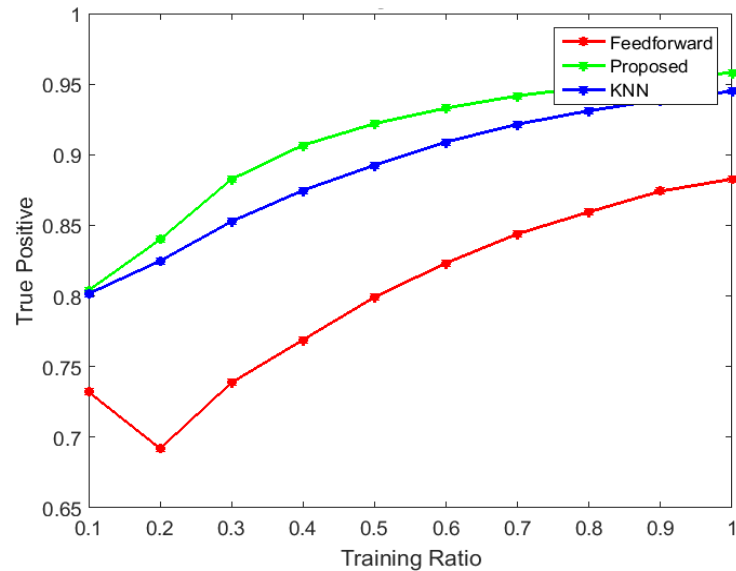

Fig 3 Comparative Analysis of True Positive

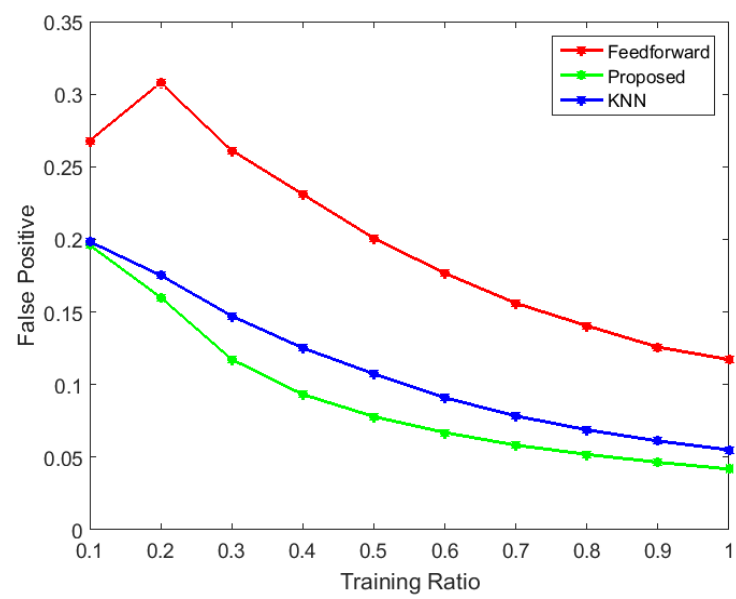

Fig 4 Comparative Analysis of False Positive

The outcome shows that proposed system offers higher true positive (Fig.3) and lower false positive in comparison to the existing classification approaches of feed forward network as well as KNN algorithm. The overall processing time of the algorithm for proposed system is found to be 2.3664 seconds whereas that of feed forward and $\mathrm{KNN}$ is found to be 7.8771 seconds and 3.1988 seconds. Therefore, this proves that the proposed system offers not only reliable solution to perform classification of the criticality situations for a given MRI image of breast cancer but also highly reduced computational complexity with faster processing time. A closer look into the outcome will also show that performance of KNN and proposed system is nearly similar however proposed system slightly outperforms KNN owing to more inclusion of learning time whereas proposed system doesn't uses training data but it uses the features extracted from it that considerably reduces processing time of proposed algorithm.

\section{CONCLUSION}

This paper has presented a novel framework that addresses the problem of classification of breast cancer with respect to criticality of the masses i.e. benign and malignant. The significant contribution of the paper are mainly two-fold as following e.g. i) a novel and simple preprocessing mechanism is presented using non-linear mapping principle which make the input DICOM image more enhance. It maintains a proper equilibrium between brightness and contrast, ii) a novel integral transformation scheme has been introduced that equally focuses on extracting two dimensional coefficient by implementing wavelet transform and it also uses watershed algorithm to support a better form of segmentation towards the gradient magnitude. This mechanism significantly assists in increasing accuracy level of true positive. Our future work will be further to investigate about evolving up with a novel morphological-based classification process which is another less explored area.

\section{REFERENCES}

[1] S. Bhattacharyya, P. Dutta, S. Chakraborty, Hybrid Soft Computing Approaches: Research and Applications, Springer, 2015

[2] "Breast Cancer Stastics", http://ww5.komen.org/BreastCancer/Statistics.html, retrieved on $16^{\text {th }}$ March 2018

[3] L. Tabar, T. Tot, Breast Cancer: Early Detection with Mammography: Crushed Stone-like Calcifications: The Most Frequent Malignant Type, Thieme, 2011

[4] B. Hela, M. Hela, H. Kamel, B. Sana and M. Najla, "Breast cancer detection: A review on mammograms analysis techniques," 10th International MultiConferences on Systems, Signals \& Devices 2013 (SSD13), Hammamet, 2013, pp. 1-6

[5] N. El Atlas, M. El Aroussi and M. Wahbi, "Computeraided breast cancer detection using mammograms: A review," 2014 Second World Conference on Complex Systems (WCCS), Agadir, 2014, pp. 626-631.

[6] D. Li, L. Wang, J. Wang, Z. Xue and S. T. C. Wong, "Transductive local fisher discriminant analysis for gene expression profile-based cancer classification," 2017 IEEE EMBS International Conference on Biomedical \& Health Informatics (BHI), Orlando, FL, 2017, pp. 49-52.

[7] P. Guillen and J. Ebalunode, "Cancer Classification Based on Microarray Gene Expression Data Using Deep Learning," 2016 International Conference on Computational Science and Computational Intelligence (CSCI), Las Vegas, NV, 2016, pp. 1403-1405.

[8] P. Qiu, Z. J. Wang and K. j. R. Liu, "Genomic processing for cancer classification and prediction - Abroad review of the recent advances in model-based genomoric and proteomic signal processing for cancer detection," in IEEE Signal Processing Magazine, vol. 24, no. 1, pp. 100-110, Jan. 2007.

[9] N. Kharel, A. Alsadoon, P. W. C. Prasad and A. Elchouemi, "Early diagnosis of breast cancer using contrast limited adaptive histogram equalization (CLAHE) and Morphology methods," 2017 8th International Conference on Information and Communication Systems (ICICS), Irbid, Jordan, 2017, pp. 120-124

[10] C. Arya and R. Tiwari, "Expert system for breast cancer diagnosis: A survey," 2016 International Conference on Computer Communication and Informatics (ICCCI), Coimbatore, 2016, pp. 1-9.

[11] A. B. Ashraf, S. C. Gavenonis, D. Daye, C. Mies, M. A. Rosen and D. Kontos, "A Multichannel Markov Random Field Framework for Tumor Segmentation With an Application to Classification of Gene Expression-Based Breast Cancer Recurrence Risk," in IEEE Transactions on Medical Imaging, vol. 32, no. 4, pp. 637-648, April 2013. 
[12] K. S. Beevi, M. S. Nair and G. R. Bindu, "A MultiClassifier System for Automatic Mitosis Detection in Breast Histopathology Images Using Deep Belief Networks," in IEEE Journal of Translational Engineering in Health and Medicine, vol. 5, no. , pp. 1-11, 2017.

[13] S. Beura, B. Majhi, R. Dash and S. Roy, "Classification of mammogram using two-dimensional discrete orthonormal S-transform for breast cancer detection," in Healthcare Technology Letters, vol. 2, no. 2, pp. 46-51, 42015.

[14] Z. Chen, H. Strange, A. Oliver, E. R. E. Denton, C. Boggis and R. Zwiggelaar, "Topological Modeling and Classification of Mammographic Microcalcification Clusters," in IEEE Transactions on Biomedical Engineering, vol. 62, no. 4, pp. 1203-1214, April 2015.

[15] K. Ganesan, U. R. Acharya, C. K. Chua, C. M. Lim and K. T. Abraham, "One-Class Classification of Mammograms Using Trace Transform Functionals," in IEEE Transactions on Instrumentation and Measurement, vol. 63, no. 2, pp. 304-311, Feb. 2014.

[16] K. Odajima and A. P. Pawlovsky, "A detailed description of the use of the $\mathrm{kNN}$ method for breast cancer diagnosis," 2014 7th International Conference on Biomedical Engineering and Informatics, Dalian, 2014, pp. 688-692.

[17] A. P. Pawlovsky and M. Nagahashi, "A method to select a good setting for the kNN algorithm when using it for breast cancer prognosis," IEEE-EMBS International Conference on Biomedical and Health Informatics (BHI), Valencia, 2014, pp. 189-192.

[18] N.Uniyal, H. Eskandari, P. Abolmaesumi, S. Sojoudi, P. Gordon, L. Warren, R. N. Rohling, S.E. Salcudean, and M. Moradi, "Ultrasound RF Time Series for Classification of Breast Lesions," in IEEE Transactions on Medical Imaging, vol. 34, no. 2, pp. 652-661, Feb. 2015.

[19] F. Ahmad, N. A. M. Isa, M. H. M. Noor and Z. Hussain, "Intelligent Breast Cancer Diagnosis Using Hybrid GAANN," 2013 Fifth International Conference on Computational Intelligence, Communication Systems and Networks, Madrid, 2013, pp. 9-12.

[20] H. Jouni, M. Issa, A. Harb, G. Jacquemod and Y. Leduc, "Neural Network architecture for breast cancer detection and classification," 2016 IEEE International Multidisciplinary Conference on Engineering Technology (IMCET), Beirut, 2016, pp. 37-41.

[21] M. G. Kanojia and S. Abraham, "Breast cancer detection using RBF neural network," 2016 2nd International Conference on Contemporary Computing and Informatics (IC3I), Greater Noida, India, 2016, pp. 363-368.

[22] S. Saini and R. Vijay, "Mammogram Analysis Using Feed-Forward Back Propagation and Cascade-Forward
Back Propagation Artificial Neural Network," 2015 Fifth International Conference on Communication Systems and Network Technologies, Gwalior, 2015, pp. 1177-1180.

[23] L. S. Solanki, S. Singh and D. Singh, "An ANN approach for false alarm detection in microwave breast cancer detection," 2016 IEEE Congress on Evolutionary Computation (CEC), Vancouver, BC, 2016, pp. 13701374.

[24] F. A. Spanhol, L. S. Oliveira, C. Petitjean and L. Heutte, "Breast cancer histopathological image classification using Convolutional Neural Networks," 2016 International Joint Conference on Neural Networks (IJCNN), Vancouver, BC, 2016, pp. 2560-2567.

[25] C.P. Utomo, A. Kardiana and R. Yuliwulandari, "Breast cancer diagnosis using artificial neural networks with extreme learning techniques", International Journal of Advanced Research in Artificial Intelligence, vol. 3(7), pp.10-14, 2014

[26] A. A. Wahab, M. I. M. Salim, J. Yunus and M. N. C. Aziz, "Tumor localization in breast thermography with various tissue compositions by using Artificial Neural Network," 2015 IEEE Student Conference on Research and Development (SCOReD), Kuala Lumpur, 2015, pp. 484-488.

[27] N. Alpaslan, A. Kara, B. Zencir and D. Hanbay, "Classification of breast masses in mammogram images using KNN," 2015 23nd Signal Processing and Communications Applications Conference (SIU), Malatya, 2015, pp. 1469-1472.

[28] S. H. Bouazza, N. Hamdi, A. Zeroual and K. Auhmani, "Gene-expression-based cancer classification through feature selection with KNN and SVM classifiers," 2015 Intelligent Systems and Computer Vision (ISCV), Fez, 2015, pp. 1-6.

[29] B. Hachemi, S. Oudjemia, F. Alim, S. Seddiki, F. Talbi and M. Abdelaziz, "Cerebral abnormalities detection by region-growing segmentation and KNN classification," Fifth International Conference on the Innovative Computing Technology (INTECH 2015), Galcia, 2015, pp. 23-26.

[30] K. Machhale, H. B. Nandpuru, V. Kapur and L. Kosta, "MRI brain cancer classification using hybrid classifier (SVM-KNN)," 2015 International Conference on Industrial Instrumentation and Control (ICIC), Pune, 2015, pp. 60-65.

[31] "TCIA Collections", http://www.cancerimagingarchive.net/, retrieved on $16^{\text {th }}$ March, 2018. 